\title{
EFICACIA DEL PROGRAMA EDUCATIVO "EXPRÉSATE" SOBRE LOS CONOCIMIENTOS DEL EMBARAZO ADOLESCENTE EN JÓVENES DE HUANCAVELICA
}

\author{
EFFECTIVENESS OF THE EDUCATIONAL PROGRAM "EXPRESS YOURSELF" ON THE KNOWLEDGE OF \\ ADOLESCENT PREGNANCY IN YOUNG PEOPLE FROM HUANCAVELICA, PERU
}

\author{
Rossibel Juana Muñoz De la Torre (iD 1,a,d,e , Luz Elizabeth Clemente-Cóndor (iD) 1,b, \\ Cintya Carbajal-Chávez (iD ${ }^{1, b}$, Edwin Jony Toral-Santillán (iD) 2,c,d
}

\begin{abstract}
Objetivo: Evaluar la eficacia del programa educativo "Exprésate" sobre el nivel de conocimiento del embarazo adolescente en las estudiantes de la Institución Educativa. Francisca Diez Canseco de Castilla, Huancavelica, Perú. Material y métodos: Estudio de diseño cuasi experimental trabajado con 205 estudiantes femeninas distribuidos en 8 secciones (A-H), una de ellas ("E") se tomó como prueba piloto conformado por 30 estudiantes, para la validación del cuestionario Pre-Test y Pos-Test a través del KR-20; luego se desarrolló sesiones secuenciales del programa educativo "Exprésate" con pre y post test. La variación de indicadores fue evaluada mediante la prueba de rangos de Wilcoxon para muestras pareadas, con un nivel de confianza del 95\%. Resultados: En el Pre-Test el 24,55\% mostró un nivel de conocimiento bajo $(5,02 \pm 1,22)$; un $74,55 \%$ medio $(9,52 \pm 1,76)$ y un $0,90 \%$ alto $(15,5 \pm 0,71)$; mientras que en el Pos-Test, realizado concluido el programa educativo, se halló que el $23,90 \%$ presentó un nivel de conocimiento medio $(13,02 \pm 1,36)$ y un $76,10 \%$ presentó un nivel alto $(16,81 \pm 1,36)$, así mismo, la variación de los valores pre y post test resultó ser estadísticamente significativa $(p<0,001)$. Conclusión: El programa educativo "Exprésate" es eficaz sobre el nivel de conocimiento del embarazo adolescente.
\end{abstract}

Palabras clave: Embarazo adolescente, Eficacia de intervención, Conocimiento (Fuente: DeCS, BIREME)

Objective: Evaluate the effectiveness of the educational program "Express yourself" on the level of knowledge of adolescent pregnancy in the students of the Educational Institution. Francisca Diez Canseco de Castilla, Huancavelica, Peru. Material and methods: Quasi-experimental design study worked with 205 female students distributed in 8 sections (AH), one of them ("E") was taken as a pilot test made up of 30 students, for the validation of the Pre-Test and Post-Test questionnaire through of the KR-20; then sequential sessions of the educational program "Express yourself" were developed with pre and posttest. The variation of indicators was evaluated using the Wilcoxon rank test for paired samples, with a confidence level of 95\%. Results: In the Pre-Test, $24.55 \%$ showed a low level of knowledge (5.02 \pm 1.22$) ; 74.55 \%$ medium (9.52 \pm 1.76$)$ and $0.90 \%$ high $(15.5 \pm 0.71)$; While in the Post-Test, carried out after the educational program, it was found that $23.90 \%$ presented a medium level of knowledge $(13.02 \pm 1.36)$ and $76.10 \%$ presented a high level $(16,81 \pm 1.36)$, likewise, the variation of the pre and posttest values turned out to be statistically significant $(p<0.001)$. Conclusion: The educational program "Express yourself" is effective on the level of knowledge of adolescent pregnancy.

Key words: Adolescent pregnancy, Effectiveness of intervention, Knowledge (Source: MeSH NLM)

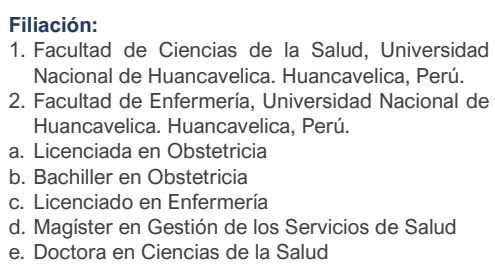

Citar como: Muñoz De la Torre R, Clemente-Cóndor LE, Carbajal-Chávez C, Toral-Santillán EJ. Eficacia del programa educativo "Exprésate" sobre los conocimientos del embarazo adolescente en jóvenes de Huancavelica. Revista Internacional de Salud Materno Fetal. 2020; 5 (3): 4-9. DOI: https://doi.org/10.47784/rismf.2020.5.3.93
Financiamiento: Autofinanciado por los autores Conflictos de interés: Los autores declaran no presentar conflictos de interés. Correspondencia: Rossibe Juana Muñoz De la Torre (rossibel.delatorre@unh.edu.pe)

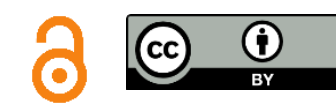

Recibido: 13 de Abril del 2020 Aprobado: 25 de Septiembre del 2020 Publicado: 30 de Septiembre del 2020 


\section{INTRODUCCIÓN}

La evolución del embarazo en la adolescente tiene importantes consecuencias sociales y de salud sobre la madre adolescente y su hijo en comparación con las gestantes adultas (1); el aspecto emocional y social que genera el impacto del embarazo es un factor de riesgo que no le permite asimilar la idea de convertirse en madre (2), por lo que requerirá del apoyo familiar para evitar evadir de la responsabilidad o provocar abortos inducidos (3); pues el embarazo adolescente trasciende la problemática de salud y termina acrecentando la pobreza junto con la exclusión de la niña y la mujer a las oportunidades de educación, empleo y participación social (4). El embarazo adolescente pudo generarse por un acto de amor, falla de método anticonceptivo, coito ocasional por consumo de alcohol o droga, por violación (5), la misma que tiene un impacto social y emocional para la madre; estos factores tienen efectos en las proyecciones de vida de la adolescente (3).

En América latina y el Caribe, el embarazo adolescente ocupa el segundo lugar a nivel mundial (6), unos dieciséis millones de mujeres entre los 15 a 19 años dan a luz cada año (7). En el Perú, el 13.5\% son embarazo adolescente siendo frecuente entre los 18 a 19 años (8), todos atribuidos a factores causales como la falta de información y acceso restringido a una educación sexual integral adecuados (5).

Estudios demuestran asociación inversa entre el nivel educativo y la fecundidad, es decir a mayor educación menor fecundidad (9); por tanto, la educación es un factor por tomar en consideración y plantear alternativas, como los programas educativos.

En el Departamento de Huancavelica se observó un incremento de casos de embarazos adolescentes, hecho que provoca deserción escolar, hogares mal constituidos y pobreza. Al respecto, la estrategia regional de salud sexual y reproductiva de la dirección regional de Salud Huancavelica, en base al sistema de información en Salud - HIS reportó que el 2015 hubo 876 embarazos adolescentes (12.7\%), mientras que el 2016 fueron 915 (13.8\%) y el 2017 se dieron 891 (13.9\%) (10); así mismo en la I.E Francisca Diez Canseco se reportó embarazo adolescente a partir de los 13 años de edad; acontecimientos que deben ser prevenidos considerando la educación como eje pilar (9).

En ese sentido, el presente estudio tuvo como objetivo evaluar la eficacia del programa educativo "Exprésate" sobre el nivel de conocimiento del embarazo adolescente cuyos resultados sirven como evidencia científica para observar como el programa educativo es eficaz sobre el nivel de conocimientos, de esta manera informar adecuadamente a las adolescentes sobre el embarazo y sus implicancias buscando la prevención de embarazo adolescente y evitando el impacto negativo que provocaría.

\section{MATERIAL Y MÉTODOS}

\section{TIPO DE ESTUDIO}

Estudio de enfoque cuantitativo, prospectivo, longitudinal y analítica, con diseño cuasi experimental.

\section{POBLACIÓN}

Correspondió a estudiantes de la Institución Educativa Francisca Diez Canseco ubicada en la ciudad de Huancavelica, Perú; la Institución educativa es de carácter público con enseñanza gratuita, donde alberga a las estudiantes femeninas de bajo recursos económicos afiliados al Seguro integral de Salud (SIS) gratuito. Los datos se recolectaron en los meses de julio a diciembre de 2018.

La población correspondió inicialmente a 250 jóvenes estudiantes, distribuidos en 8 aulas $(\mathrm{A}-\mathrm{H})$ de ellas se seleccionó aleatoriamente a 30 jóvenes de un solo aula para desarrollar la prueba piloto, además de 15 estudiantes que fueron excluidas por falta de continuidad en el programa; por tanto, la muestra fue de 205 jóvenes, todas cursando el $5^{\circ}$ grado del nivel secundario que aceptaron participar y estuvieron presentes desde la aplicación del pre test en la apertura del programa educativo con participación activa en las 6 sesiones educativas y finalmente en la aplicación del post test, todos los procesos con un intervalo de 15 días y las sesiones con una duración de 45 minutos..

\section{VARIABLES}

Se midieron los datos sociodemográficos como edad y grupo familiar; respecto al nivel de conocimientos sobre el embarazo adolescente la variable tuvo tres niveles alto, medio y bajo. Para la 
variable eficacia del programa educativo "exprésate" se midieron el desarrollo de las sesiones en temas como adolescencia, embarazo, autoestima y sexualidad, riesgos y complicaciones del embarazo adolescente y proyecto de vida.

\section{TÉCNICAS Y PROCEDIMIENTOS}

Se empleó la encuesta para la administración del pre y post test sobre el embarazo adolescente y la entrevista estructurada para la determinación de los datos sociodemográficos.

La prueba estuvo conformada por 18 preguntas de alternativas múltiples parala determinación del nivel de conocimientos sobre embarazo adolescente, validado a través del juicio de expertos y la confiabilidad mediante la psicometría KuderRichardson (KR-20) siendo aceptables.

El Programa educativo Exprésate" es una herramienta preventiva adecuada en el modelo Pedagógico cognitivo conductual y la teoría de aprendizaje significativo por recepción de Ausubel, en la que se desarrolló seis sesiones educativas organizadas y secuenciales, orientadas a la prevención del embarazo.

Para la recolección de datos, se solicitó la autorización a la directora de la Institución Educativa Francisca Diez Canseco de Castilla quien se entrevistó con los padres de familia para la difusión del estudio, luego se participó de la reunión donde nos facilitó el registro de estudiantes matriculados y se estableció horarios de tutoría para el desarrollo de las sesiones. Se apertura el programa educativo con el consentimiento de las estudiantes y la aplicación del pre test, una vez entrevistadas sobre los aspectos sociodemográficos se da inicio al desarrollo de las seis sesiones educativas con permanencia de todas las estudiantes por un espacio de 45 minutos organizados en 7 aulas en horarios diversos con encuentros quincenales; el desarrollo de las sesiones estuvo dirigida por la obstetra, apoyada por el equipo de investigación quienes guiaron las actividades acorde a lo validado en el programa educativo

\section{ANÁLISIS DE DATOS}

Se elaboró una base de datos en el programa estadístico SPSS para Windows v.25, con la finalidad de realizar el análisis estadístico descriptivo e inferencial de acuerdo con la naturaleza del estudio. En las variables sociodemográficas se aplicó la distribución de frecuencias y porcentajes. Para evaluar la eficacia del programa educativo "exprésate" sobre el nivel de conocimientos del embarazo adolescente se aplicó las tablas de contingencia, la prueba de rangos pareados de Wilcoxon con un IC 95\% y un nivel de significancia estadística $p<0,05$.

\section{ASPECTOS ÉTICOS}

El estudio fue aprobado por el Comité de Ética de la Facultad de Ciencias de la Salud de la Universidad Nacional de Huancavelica con carta $\mathrm{N}^{\circ} 15-2018-\mathrm{D}$ FCS- VRAC/UNH.

\section{RESULTADOS}

El $73,7 \%$ de las estudiantes tenían 16 años, en cuanto al grupo familiar el $64,4 \%$ era nuclear, seguido del 20,5\% monoparental, el 12,2\% familia ampliada y el 2,9\% equivalente y la distribución de estudiantes por aula fueron proporcionales (Tabla 1).

Tabla 1. Datos sociodemográficos de las jóvenes estudiantes

\begin{tabular}{lcc}
\hline & $\mathbf{n}$ & $\%$ \\
\hline Distribución de aulas & 28 & 13,7 \\
Aula A & 30 & 14,6 \\
Aula B & 29 & 14,1 \\
Aula C & 27 & 13,2 \\
Aula D & 30 & 14,6 \\
Aula F & 31 & 15,1 \\
Aula G & 30 & 14,6 \\
Aula H & & \\
Edad & 34 & 16,6 \\
15 años & 151 & 73,7 \\
16 años & 19 & 9,3 \\
17 años & 1 & 0,5 \\
18 años & & \\
Grupo familiar & 132 & 64,4 \\
Nuclear & 42 & 20,5 \\
Monoparental & 25 & 12,2 \\
Ampliada & 6 & 2,9 \\
Equivalente & $\mathbf{2 0 5}$ & 100,0 \\
& & \\
\hline
\end{tabular}

El nivel de conocimientos sobre embarazo adolescente en el Pre-Test del programa educativo "Exprésate" fue medio con el $74,6 \%$ y una nota promedio de 9.5 , seguido del nivel bajo con el $24,4 \%$, 
revistamaternofetal.com

promedio de 5 y nivel alto con el $0,98 \%$, un promedio de 15,5 (Tabla 2).

Tabla 2. Nivel de conocimiento sobre el embarazo adolescente post intervención del programa educativo "Exprésate"

\begin{tabular}{|c|c|c|c|c|}
\hline & $n$ & $\%$ & Media & D.S \\
\hline \multicolumn{5}{|l|}{$\begin{array}{l}\text { Nivel de } \\
\text { conocimiento }\end{array}$} \\
\hline Bajo & 50 & 24,39 & 5,02 & 1,22 \\
\hline Medio & 153 & 74,63 & 9,52 & 1,76 \\
\hline Alto & 2 & 0,98 & 15,5 & 0,71 \\
\hline Total & 205 & 100 & & \\
\hline
\end{tabular}

D.S: Desviación estándar

El nivel de conocimientos sobre embarazo adolescente en el Pos-Test, evaluada quince días después de concluido el programa educativo fue alto en el $76,1 \%$ con promedio de 16,8 , seguido del nivel medio $23,9 \%$ con un promedio de 13 (Tabla 3 ).

Tabla 3. Nivel de conocimiento sobre el embarazo adolescente post intervención del programa educativo "Exprésate"

\begin{tabular}{|c|c|c|c|c|}
\hline & $n$ & $\%$ & Media & D.S \\
\hline \multicolumn{5}{|l|}{$\begin{array}{l}\text { Nivel de } \\
\text { conocimiento }\end{array}$} \\
\hline Bajo & 0 & 0 & - & - \\
\hline Medio & 49 & 23,9 & 13,02 & 1,35 \\
\hline Alto & 156 & 76,10 & 16,81 & 1,36 \\
\hline Total & 205 & 100 & & \\
\hline
\end{tabular}

El desarrollo del programa educativo "exprésate" logró incrementar el nivel de conocimientos sobre el embarazo adolescente; donde el pretest mostró participantes con conocimiento bajo, mientras que al final gran parte de la muestra mostró un conocimiento alto; ambos momentos sometidos a una prueba de rangos de Wilcoxon para muestras pareadas mostró una variación estadísticamente significativa $(p<0,05)$. (Tabla 4).

\section{DISCUSIÓN}

El embarazo adolescente es un tema que trasciende los ámbitos de la salud física y psicosocial, revelan el debilitamiento del tejido social fruto de la descomposición de relaciones sociales y comunitarias.

Tabla 4. Eficacia del programa educativo "Exprésate" sobre el nivel de conocimiento del embarazo adolescente

\begin{tabular}{lccccc}
\hline & \multicolumn{2}{c}{ Pre-Test } & \multicolumn{2}{c}{ Post-Test } & $\mathrm{p}^{\dagger}$ \\
\cline { 2 - 4 } & $\mathrm{n}$ & $\%$ & $\mathrm{n}$ & $\%$ & \\
\hline $\begin{array}{l}\text { Nivel de } \\
\text { conocimiento }\end{array}$ & & & & & \\
Bajo & 50 & 24,39 & 0 & 0,0 & \\
Medio & 153 & 74,63 & 49 & 23,90 & $<0,001$ \\
Alto & 2 & 0,98 & 156 & 76,10 & \\
\hline
\end{tabular}

† Evaluado mediante la prueba de rangos pareados de Wilcoxon

El $73,7 \%$ de las jóvenes estudiantes tienen edades de 16 años; el 64,4\% viven con los padres y hermanos; al respecto se enfatiza que las adolescentes tardías tienen mayor riesgo psicosocial ante un embarazo precoz, el inicio sexual temprano se asocia a factores de vulnerabilidad y que la familia es como una institución socializadora y formadora de sus miembros; sin embargo, estos aspectos están deteriorados por multiplicidad de factores culturales, humanos, económicos y tradicionales (11), al respecto Castillo (12) encontró que el $96,7 \%$ de los padres de familia tienen un nivel bajo de conocimientos sobre educación sexual influenciando de forma negativa en la orientación de los hijos.

En el estudio el nivel de conocimientos sobre embarazo adolescente obtenidos del Pre test fue de nivel medio de $74,6 \%$ con un promedio de 9,5 seguido del nivel bajo con el $24,3 \%$ con promedio de 5 , identificando desconocimiento sobre el tema; similares resultados se encontraron en diferentes países (13-15), enfatizando que muchos de los casos el embarazo adolescente está asociado a condiciones de pobreza y marginación donde vive una mujer, en donde el aspecto crítico es la educación, en el Perú las mujeres tienen menor oportunidad de recibir educación a diferencia de los varones; el vínculo directo entre la pobreza, poca educación, baja autoestima, dependencia económica y actualmente la alineación en el abordaje adolescente es inverso. El desconocimiento en esta materia está relacionado con las creencias y estereotipos que tienen la familia y comunidad con la idea de tocar que abordar el tema es propio de despertar intereses sexuales tempranos. 
Al respecto, Menkes y Suarez (16) enfatizan en la importancia de crear programas educativos orientados a la prevención del embarazo adolescente y así prevenir indirectamente las complicaciones clínicas que usualmente padecen como la hipertensión del embarazo, anemia, infecciones, parto prematuro, bajo peso al nacer y cesáreas (17), y en un esfuerzo sintetizador se afirma que no solo tiene implicancia clínica en la madre sino también en sus eventuales hijos y en su grupo familiar (18).

El desarrollo del programa educativo Exprésate fue eficaz sobre el nivel de conocimientos del embarazo adolescente con una significancia estadística menor a 0,05 ; además las estudiantes mejoraron el nivel de conocimientos donde el $76,1 \%$ obtuvo un nivel alto, seguido del $23,9 \%$ de nivel medio; estos resultados fueron menores a lo hallados por Cercado y otros (19), diferencias que se deben a la cantidad de estudiantes intervenidas, cuanto más pequeño es el grupo intervenido la educación se muestra personalizados con mejores resultados. Se enfatiza la necesidad de continuar con el desarrollo de programas educativos en la etapa escolar sin reemplazar el rol de los padres con el fin de alcanzar el desarrollo en la toma de decisiones y comunicación asertiva.

Se concluye que intervenir con el programa educativo mejoró los niveles de conocimientos de las jóvenes estudiantes y similar a lo demostrado en varios estudios (20) se espera el retraso del inicio de la actividad sexual, provisión de información y evitar el embarazo adolescente.

Frente a los resultados hallados se recomienda al profesional de la salud promover la aplicación de programas educativos dirigidos a las y los jóvenes haciendo uso de las tecnologías de comunicación e información forma periódica adaptados a diversos escenarios. 


\section{REFERENCIAS}

1. Posada C. Embarazo en la adolescencia: no una opción, sino una falta de opciones. Revista Sexología y Sociedad. 2014;10(25). [Link]

2. Instituto Nacional de Estadística e Informática. Indicadores de Resultados de los Programas Presupuestales, Primer Semestre 2018, Perú. [Internet] Lima: INEI. Julio. 2018.

3. Llanos ES, Miniet AML. Factores que influyen en el embarazo de adolescentes. FACSALUD-UNEMI. 2019;3(4):36-42. [Link]

4. Gálvez Henry F, Rodríguez Sánchez BA, Lugones Botell M, Altunaga Palacio M. Características epidemiológicas del embarazo en la adolescencia. Revista Cubana de Obstetricia y ginecología. 2017;43(3):15-27. [Link]

5. Bendezú G, Espinoza D, BendezúQuispe G, Torres-Román JS, HuamánGutiérrez RM. Características y riesgos de gestantes adolescentes. Revista peruana de Ginecología y Obstetricia. 2016;62(1):13-8. [Link]

6. Organización Panamericana de la Salud. América Latina y el Caribe tienen la segunda tasa más alta de embarazo adolescente en el mundo. [Internet] Washington: OPS. [Link]

7. El fondo de las naciones unidas para la infancia (UNICEF). Acelerar el progreso hacia la reducción del embarazo en la adolescencia en América Latina y el Caribe. Washington, DC: Unicef. 2016.
8. Instituto Nacional de Estadística e Informática-Perú.

Embarazo adolescente. [Internet] Lima: INEI. 2017.

9. Seperak Viera RA, Rivera Calcina R Determinantes sociodemográficos de la alta fecundidad en mujeres peruanas. Revista Chilena de Obstetricia y Ginecología. 2018;83(5):452-63. [Link]

10. Epidemiología. Embarazo adolescente. Dirección Regional de Salud Huancavelica; 2017

11. Afán-González A. La diversidad afectivosexual en Educación Primaria. Universidad de Sevilla. Trabajo de fin de grado. 2018. [Link]

12. Castillo Tapullima EM. Efectividad de Programa de Educación Sexual en el conocimiento sobre sexualidad de los padres de familia del nivel secundario de $1^{\circ}-5^{\circ}$ de la IE 0288 Pachilla, juliodiciembre 2017. Universidad Naciona de San Martín [Tesis de grado] 2020. [Link]

13. Leal I, Luttges C, Troncoso P, Leyton C Molina T, Eguiguren P. Conocimientos y práctica clínica de los proveedores de salud para la prevención del embarazo adolescente según marco legal chileno. Revista Médica de Chile. 2016;144(5):577-84. [Link]

14. Salazar Avalos LR. Programa educativo "Yo me cuido" y su relación con e incremento del nivel de conocimientos sobre prevención de embarazo adolescente I.E 7078 "El Nazareno". Universidad Nacional del Santa [Tesis de post grado] 2018. [Link]
15. D'Añari-Cabrera JR. Factores Biosociodemograficos asociados al embarazo precoz en adolescentes gestantes atendidas en el HRHDE, AbrilMayo 2019. Universidad Nacional de San Agustín de Arequipa [Tesis de grado]. 2019. [Link]

16. Menkes C, Suárez L. Sexualidad y embarazo adolescente en México. Papeles de población. 2003;9(35):23362. [Link]

17. Guerrero GEM, Cabrera IN, Rodríguez $\mathrm{YH}$, Castellanos JE. Pregnancy and its complications in adolescent mothers. Revista Cubana de Obstetricia y Ginecología. 2012;38(3). [Link]

18. Gutiérrez Sánchez MC, Reyes Céspedes AN, Villalobos Hernández NE. Participación comunitaria educativa en el diseño de un programa de prevención de embarazo adolescente mediante la IAP en la localidad de San Cristobal Sur en Bogotá. Universidad Cooperativa de Colombia [Investigación dirigida]. 2016. [Link]

19. Cercado Esteban VP, Castillo Quispe JN. Efectividad del programa educativo conociendo mi sexualidad en el nivel de conocimientos sobre sexualidad en adolescentes. Universidad Nacional de Trujillo [Tesis de grado]. 2019. [Link]

20. Elick JH. The identification of some risk factors that may contribute to teenage pregnancy. [Internet]. 2003 